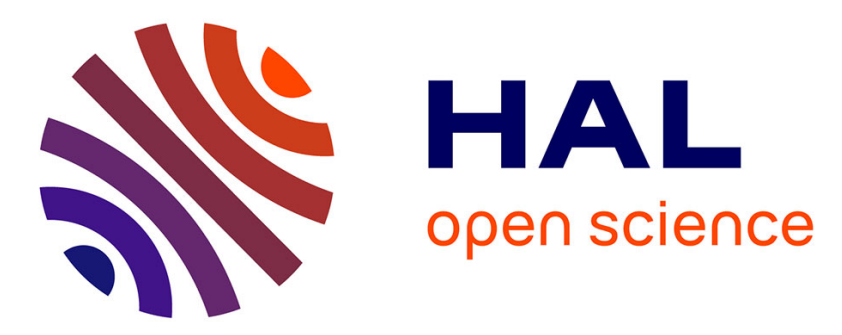

\title{
Assisted Decision-Making for Assembly Technique Selection and Geometrical Tolerance Allocation
}

Loïc Andolfatto, François Thiébaut, Claire Lartigue, Marc Douilly

\section{To cite this version:}

Loïc Andolfatto, François Thiébaut, Claire Lartigue, Marc Douilly. Assisted Decision-Making for Assembly Technique Selection and Geometrical Tolerance Allocation. 23rd CIRP Design Conference, Mar 2013, Bochum, Germany. pp.315 - 324, 10.1007/978-3-642-30817-8_31 . hal-01094429

\section{HAL Id: hal-01094429 \\ https://hal.science/hal-01094429}

Submitted on 12 Dec 2014

HAL is a multi-disciplinary open access archive for the deposit and dissemination of scientific research documents, whether they are published or not. The documents may come from teaching and research institutions in France or abroad, or from public or private research centers.
L'archive ouverte pluridisciplinaire HAL, est destinée au dépôt et à la diffusion de documents scientifiques de niveau recherche, publiés ou non, émanant des établissements d'enseignement et de recherche français ou étrangers, des laboratoires publics ou privés. 


\title{
Assisted Decision-making for Assembly Technique Selection and Geometrical Tolerance Allocation
}

\author{
Loïc Andolfatto ${ }^{12}$, François Thiébaut ${ }^{23}$, Claire Lartigue $^{23}$, and Marc Douilly ${ }^{1}$ \\ ${ }^{1}$ EADS Innovation Works, 12 rue Pasteur, 92150 Suresnes, France \\ ${ }^{2}$ LURPA, ENS de Cachan, 61 avenue du Président Wilson, 94235 Cachan Cedex, France \\ 3 IUT de Cachan, 9 Avenue de la Division Leclerc, 94235 Cachan Cedex, France \\ loic.andolfatto@ \{eads.net, lurpa.ens-cachan.fr\}
}

\begin{abstract}
Assembly process planning involves many aspects from geometrical matters to operational research. Though, the literature shows very few works about assembly technique selection.

This paper deals with an original method to select assembly techniques and to allocate component geometrical tolerances in order to minimize the product cost and to maximize the conformity rate associated with the assembly plan.

The data structures used to define a parametric assembly plan is detailed. This data structure is used to formulate a multi-objective optimization problem reflecting the concerns of the study.

The entire method is illustrated trough a case study. The results obtained are presented and followed by a discussion about the potential benefits of its application in an industrial context. The useful support that this method can provide to decision-making is highlighted. Its shared point of view from product designers to manufacturing process designers makes it an efficient tool for concurrent engineering.
\end{abstract}

Keywords: Assembly process planning, assembly technique selection, geometrical tolerance allocation, multi-objective optimization, concurrent engineering

\section{Introduction}

\subsection{Design of an Assembly Process Plan}

The assembly of large mechanical structures, such as aeronautical ones, may account for a large share of their total delivery cost. Boothroyd and Dewhurst stressed out the importance for manufacturing companies to assess a product's design by designing assembly process plan as soon as ${ }^{1}$ in order to reach the maximum performance [1]. Computeraided assembly process planning has been the subject of many research works generally aiming at finding the minimum lead time and/or cost. The performance of the assembly process plans is evaluated according to several indicators such as, for example, tooling needs, reorientations of sub-assemblies, technological similarities in consecutive operations and so on [2].

\footnotetext{
${ }^{1}$ What is called design for manufacturing and assembly (DFMA).
} 


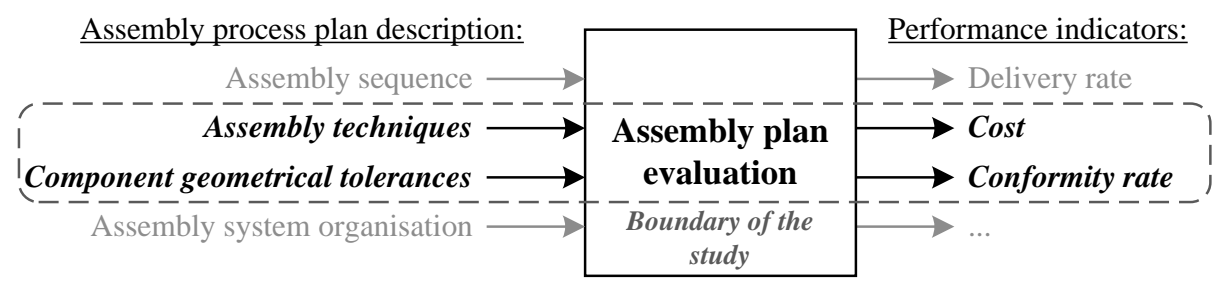

Fig. 1. Schematic view of the description and evaluation of an assembly process plan with the proposed approach boundary

As summarized in Fig. 1Schematic view of the description and evaluation of an assembly process plan with the proposed approach boundaryfigure.1.1, the description of an assembly process plan can be split into an assembly sequence, the assembly technique selected to make each attachment of the product, the geometrical tolerances allocated to each component and the description of the assembly system ${ }^{2}$. Most of the works presented in the literature only focus on specific aspects of the problem.

Assembly sequence planning, either defined as the part introduction ordering [3-5] or as the joint realization ordering [6,7], has been widely studied in the literature [2]. The joint-based approach is close to the activity-based approach proposed by Cao and Sanderson [8], and reflects some of the issues considered for the organisation of the assembly system commonly treated in operational research [9].

But the generation of assembly sequences and the organisation of the assembly system are not the only activities encapsulated in assembly process planning. Assembly technique $^{3}$ selection [10] is seldom addressed in the literature even though it may have the greatest impact on production cost, according to Abdullah et al. [11].

Selecting assembly techniques implies setting the associated geometrical capabilities. The concern of geometrical quality of the assembled product (commonly called tolerancing) is therefore coupled with the assembly process planning. Up to this point, the geometrical tolerances allocated to the components to be assembled may be part of the information contained in a comprehensive assembly process plan $[7,12,13]$.

\subsection{Proposed Approach}

Massive automation has been the key solution to answer the need for decreasing manufacturing cost and increasing delivery rate in many manufacturing domains. But in the field of aeronautical structure assembly, the complexity of some attachments, the high level of geometrical requirements and the high working area required are several hurdles to an efficient use of automation with respect to manual assembly. Trades-off have to be made.

This paper aims at proposing an original method to select assembly techniques and to allocate component geometrical tolerances in order to minimize the product cost

\footnotetext{
${ }^{2}$ Either spatial or temporal.

${ }^{3}$ Also called assembly process by some authors.
} 
and to maximize the conformity rate associated with the assembly plan. The assembly sequence is assumed to be already defined and the organisation of the assembly system is not considered.

Section 2Optimization problemsection.1.2 details how an assembly process plan can be described according to a set of decision variables and how it can be evaluated according to several performance indicators. This formal view of the problem serves the definition and the resolution of a multi objective optimization problem.

A simple use case is presented in Sect. 3Use casesection.1.3 and the results obtained are detailed in Sect. 4Resultssection.1.4. Section 5Conclusionsection.1.5 concludes on the potential benefits of this approach in a concurrent engineering context.

\section{Optimization problem}

\subsection{Multi-objective Optimization}

Considering a given product and a given assembly sequence, the purpose of the presented work is to propose a method to find a set of assembly techniques and a set of geometrical tolerances that minimize the non-conformity rate and the delivery cost of a product at the same time.

A mathematical expression of this problem is given in (1Multi-objective Optimizationequation.1.2.1), where $\boldsymbol{x}$ is a decision variable vector representing an assembly plan, $X$ is the set of feasible assembly plans and $f$ is a function associating a fitness to an assembly plan. The construction of the decision variable vector $\boldsymbol{x} \in X$ is later described in Sect. 2.2Parametric Assembly Plansubsection.1.2.2.

$$
\inf \{f(\boldsymbol{x}): \boldsymbol{x} \in X\}
$$

In the present case, the fitness function $f$ must cope with several objectives (nonconformity and cost). Moreover, these objectives are likely to conflict with each other. A common solution to this issue is to combine the objectives in a single-valued fitness function. But it can prove complicated to model the actual objectives into a single performance indicator, which results in making trades-off a priori.

Solving a multi objective optimization problem seems to be more appropriate within the industrial context. From the assembly process planner point of view, an interesting point $\boldsymbol{x}^{*}$ describes an assembly process plan for which one can not find a solution that provides both lower non-conformity rate and lower cost at the same time, as pictured in Fig. 2Non-dominated assembly process plans (circles) among a population (squares) considering the simultaneous minimization of the non-conformity rate and the minimization of the delivery costfigure.1.2.

Assuming $f=[\operatorname{NCR}(\boldsymbol{x}), \mathrm{C}(\boldsymbol{x})]$, non-conformity rate function and delivery cost function described in Sect. 2.3Assembly Plan Evaluationsubsection.1.2.3, a solution of the problem given in (1Multi-objective Optimizationequation.1.2.1) is a point $\boldsymbol{x}^{*}$ satisfying (2Multi-objective Optimizationequation.1.2.2), $\boldsymbol{x}^{*}$ is called a non-dominated point.

$$
\nexists \boldsymbol{x} \in X, \operatorname{NCR}(\boldsymbol{x})<\operatorname{NCR}\left(\boldsymbol{x}^{*}\right) \text { and } \mathrm{C}(\boldsymbol{x})<\mathrm{C}\left(\boldsymbol{x}^{*}\right)
$$




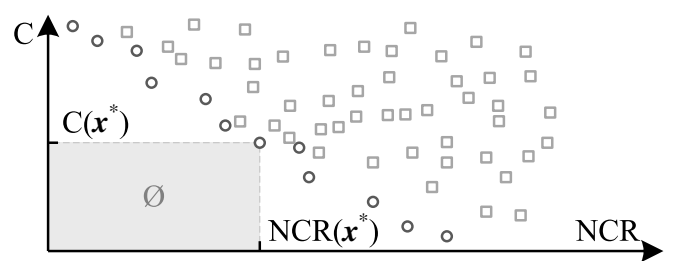

Fig. 2. Non-dominated assembly process plans (circles) among a population (squares) considering the simultaneous minimization of the non-conformity rate and the minimization of the delivery cost

The underlying mathematical problem of this work consists in finding the set of non-dominated points (or a sufficient amount of points if this set is not finite). The assembly process planner finally has to select the plan that provides the best trade-off among the set of non-dominated points a posteriori. The high level, difficult-to-make, decision appears with the rough results in hand instead of during the modelling phase required to build a black-box optimizer. Modelling every single actual objective of the problem alone is likely to be an easier task.

\subsection{Parametric Assembly Plan}

Generalities. Running the optimization problem presented in (1Multi-objective Optimizationequation.1.2.1) requires to convert the assembly process plan description known in technical terms into a mathematical vector $\boldsymbol{x}$ called assembly plan vector in the paper.

A product is a set of components linked together through joints during the assembly process. Each component is subject to geometrical variations bounded by geometrical tolerances. Each joint is made using an assembly technique. The requirement on the assembly plan vector $\boldsymbol{x}$ can be deduced out of those three assertions. It must include elements representing the technique selected for each joint of the product and elements representing the components geometrical deviations.

Assembly techniques. Considering a product with $N_{\mathrm{j}}$ joints, the $N_{\mathrm{j}}$ first elements of $\boldsymbol{x}$ are dedicated to describe which assembly technique is associated to each of the $N_{\mathrm{j}}$ joints. Assembly techniques are stored and indexed in a library with several attributes: index, associated list of assembly operations, list of costing information, geometrical capabilities, etc.

The technique assigned to the joint $j$ is the one the index of which equals the value of the $j$ th element of $\boldsymbol{x}$, as pictured in Fig. 3Translation of an assembly process plan into a decision variable vector $x$ figure.1.3.

The assembly process planner has to define the list of techniques suitable to make each joint of the product. Some additional constraints can be set among those $N_{\mathrm{j}}$ first elements: two joints can be forced to be made with the same technique for example. This reduces the set $X$ of feasible assembly plans to the technically admissible ones. 


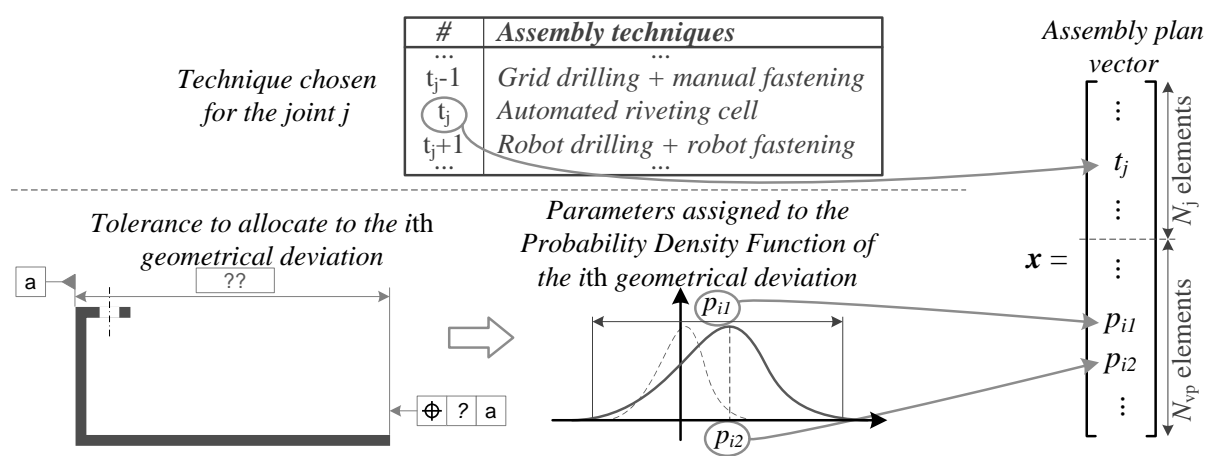

Fig. 3. Translation of an assembly process plan into a decision variable vector $\boldsymbol{x}$

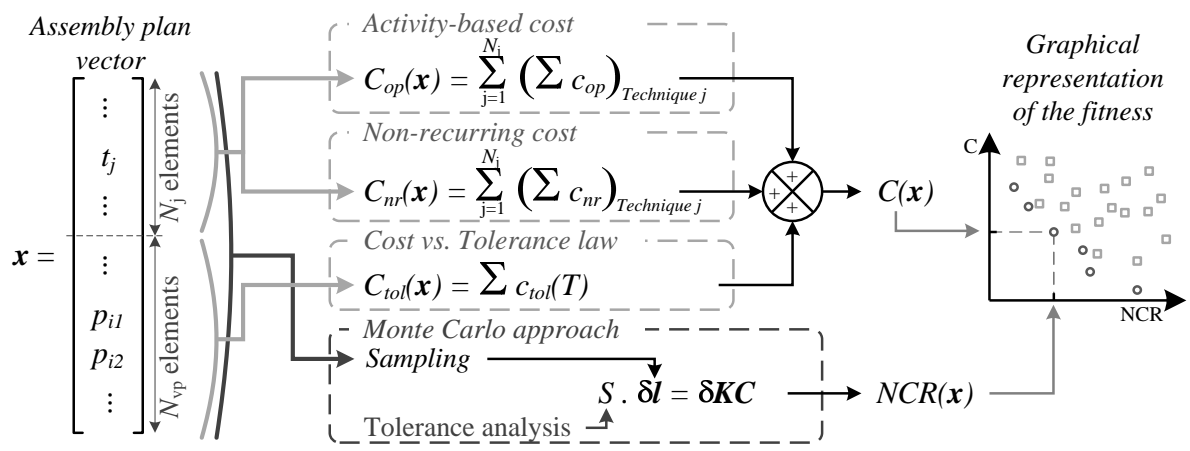

Fig. 4. Evaluation scheme and fitness representation of an assembly plan defined by its mathematical representation $\boldsymbol{x}$.

Geometrical variations and geometrical tolerances. Assuming that the probability distribution of each geometrical variation is known, allocating tolerances amounts to setting these distribution parameters, e.g. the lower bound and upper bound for a uniform probability distribution or the mean value and the standard deviation for a normal distribution. This is illustrated in Fig. 3Translation of an assembly process plan into a decision variable vector $\boldsymbol{x}$ figure.1.3. If the product's components have $N_{\mathrm{gv}}$ geometrical variations, and each of them has $k$ distribution parameters, then the assembly plan vector $\boldsymbol{x}$ also has $N_{\mathrm{vp}}=\sum_{i=1}^{N_{\mathrm{gv}}} k_{i}$ elements to describe the geometrical tolerances allocated.

Intrinsic constraints exist among those $N_{\mathrm{vp}}$ elements, such as a lower bound smaller than an upper bound for instance. Extrinsic constraints can also be declared by the user to define other technical limits, such as the minimum size of a tolerance zone. This also reduces the set $X$ of feasible assembly plans. 


\subsection{Assembly Plan Evaluation}

Non-Conformity Rate. The conformity of a product can be assessed by verifying that some of its characteristics - called key characteristics (KC) in [7] - are kept within a requirement domain defined during the functional analysis. The Non-Conformity Rate of a product is defined as the probability for a product to have at least one of its $\mathrm{KC}$ outside of its requirement domain. In this work, only geometrical $\mathrm{KC}$ are considered. Tolerancing studies commonly provide a sensitivity matrix $S$ to link the geometrical deviations $\delta \boldsymbol{l}^{4}$ to the KC deviations $\delta \boldsymbol{K} \boldsymbol{C}$ as in (3Non-Conformity Rateequation.1.2.3) $[12,14,13]$.

$$
S \cdot \delta l=\delta K C
$$

Samples of $\boldsymbol{\delta} \boldsymbol{l}$ vectors can be associated to an assembly plan vector using both PDFs of the component geometrical variations and assembly technique capabilities. A Monte Carlo approach is finally used to associate the non-conformity rate $N C R(\boldsymbol{x})$ to the assembly plan described by $\boldsymbol{x}$.

Delivery Cost. The delivery cost can be split into two cost sources: the cost associated to the assembly operations and the cost resulting from the allocation of geometrical tolerances.

Each assembly technique is characterized by an interdependent list of assembly operations (an example is given in Table 3Details of the technique Positioning with a robot as tooltable.1.3). Each operation has a fixed $\operatorname{cost} c_{\mathrm{f}}$ representing consumables and unitary tool wear for example. It also uses several resources (of hourly rate $h r_{i}$ ) during a certain amount of time $t_{\mathrm{op}}$. The elementary cost of an assembly operation is defined in (4Delivery Costequation.1.2.4):

$$
c_{\mathrm{op}}=c_{\mathrm{f}}+t_{\mathrm{op}} \times \sum_{i} h r_{i}
$$

The total activity-based cost $C_{\mathrm{op}}$ is the sum of the elementary cost of each operation, the list of which is derived from the list of selected assembly techniques given by $\boldsymbol{x}$.

The non-recurring cost per product $C_{\mathrm{nr}}$, due to the acquisition cost of all the resources required for the assembly divided by the presumable amount of product to be assembled, is also included to the total delivery cost. As the list of the resources required to assemble the product depends on the assembly technique selection, $C_{\mathrm{nr}}$ is a function of $x$.

The cost associated to a geometrical tolerance allocated is modelled by (5Delivery Costequation.1.2.5) (adapted from [15]). $T$ is the size of the tolerance interval allocated, $T_{\mathrm{lim}}, a, b, m$ and $k$ are function parameters identified according to experimental data.

$$
c_{\mathrm{tol}}(T)=a+b \cdot \mathrm{e}^{-m\left(T-T_{\mathrm{lim}}\right)} \cdot\left(T-T_{\lim }\right)^{-k}
$$

\footnotetext{
${ }^{4}$ Gathering both geometrical deviations of the components and deviations due to the assembly techniques employed.
} 
The total $\operatorname{cost} C_{\mathrm{tol}}$ associated to the geometrical tolerances allocated to the product's components is the sum of all the elementary costs evaluated thanks to (5Delivery Costequation.1.2.5) according to the tolerances described by the vector $\boldsymbol{x}$.

Finally, the sum of $C_{\mathrm{op}}, C_{\mathrm{nr}}$ and $C_{\mathrm{tol}}$ provides the delivery cost $C(\boldsymbol{x})$ associated to an assembly plan, as depicted in Fig. 4Evaluation scheme and fitness representation of an assembly plan defined by its mathematical representation $x$ figure.1.4.

\section{Use case}

\subsection{Product to be assembled}

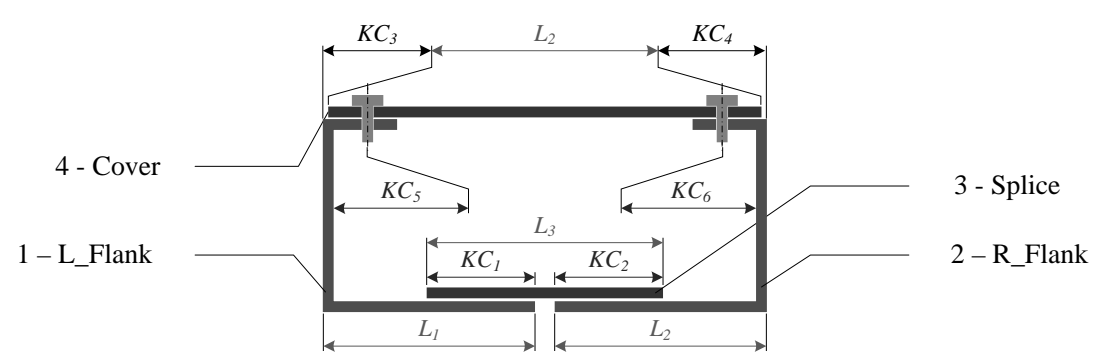

Fig. 5. Details of the product's key characteristics $\left(K C_{i}\right)$, components and their characteristics considered as subject to geometrical variation $\left(L_{j}\right)$
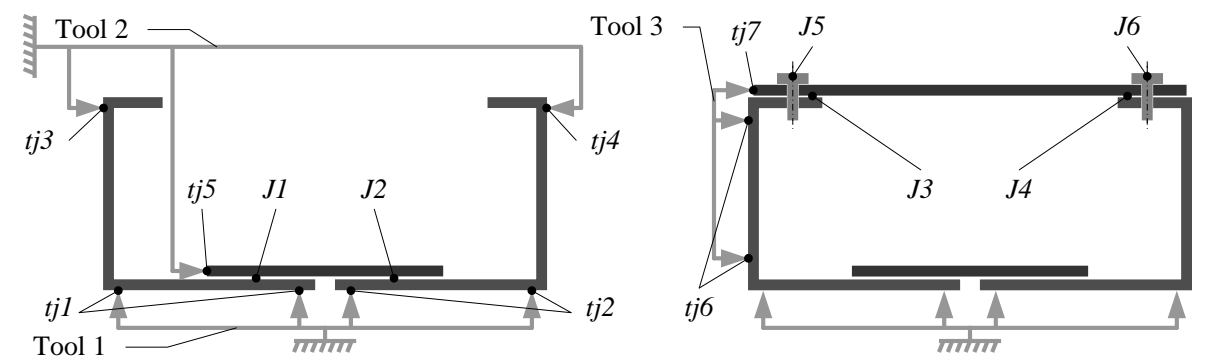

Assembly sequence: $t j 1, t j 3, t j 2, t j 4, t j 5, J 1-J 2, t j 6, t j 7, J 3-J 4, J 5, J 6$

Fig. 6. Temporary components used during assembly (referred to as Tool $k$ ), joints of the product and assembly sequence

The method described in the previous section is applied to the assembly of a simple mechanical structure composed of four components (see Fig. 5Details of the product's 
Table 1. Parameters of the Cost vs. Tolerance law defined by (5Delivery Costequation.1.2.5)

\begin{tabular}{llllll}
\hline & $\begin{array}{l}T_{\text {lim }} \\
(\mathrm{mm})\end{array}$ & $a$ & $b$ & $m$ & $k$ \\
& $($ cost unit $)$ & $($ cost unit $/ \mathrm{mm})$ & $\left(\mathrm{mm}^{-1}\right)$ & \\
\hline$L_{1}$ and $L_{2}$ & 0.01 & 0 & 200 & 1 & 1 \\
$L_{3}$ and $L_{4}$ & 0.01 & 0 & 200 & 1 & 1 \\
\hline
\end{tabular}

key characteristics $\left(K C_{i}\right)$, components and their characteristics considered as subject to geometrical variation $\left(L_{j}\right)$ figure.1.5). Three additional temporary components are also used during the assembly, as exposed in Fig. 6Temporary components used during assembly (referred to as Tool $k$ ), joints of the product and assembly sequencefigure.1.6 in which the assembly sequence is also given (as the order in which the joints are made).

The geometrical variation propagation problem is reduced to a one-dimensional study with six key characteristics and four component's dimensions subject to geometrical variations. The capabilities of the technique selected for the joints $t j 3$ to $t j 7$ are also impacting the conformity rate as these joints are positioning joints involved in the $\mathrm{KC}$ values. Deviations on $K C_{1}$ and $K C_{2}$ must be kept within $\pm 0.6 \mathrm{~mm}$. The value for $K C_{4}$ to $K C_{6}$ is $\pm 0.3 \mathrm{~mm}$.

The parameters of the cost vs. tolerance law defined by (5Delivery Costequation.1.2.5) are given in Table 1Parameters of the Cost vs. Tolerance law defined by (5Delivery Costequation.1.2.5)table.1.1. The sensitivity matrix $S$ defined in (3Non-Conformity Rateequation.1.2.3) is not detailed in the paper.

\subsection{Assembly technique library}

The assembly technique library is not entirely described in this paper but the Table 2List of the assembly techniques available associated to the joints they can be used fortable.1.2 presents the techniques considered. The Table 3Details of the technique Positioning with a robot as tooltable.1.3 details the information stored in the library for the Positioning with a robot as tool technique. In addition to the feasibility constraints detailed in Table 2List of the assembly techniques available associated to the joints they can be used fortable.1.2, the joints $t j 3$ to $t j 5$ must be done with the same technique. So do the joints $t j 6$ and $t j 7$.

\section{Results}

The optimization problem defined in (1Multi-objective Optimizationequation.1.2.1) is solved using the Non-Sorting Genetic Algorithm II (implemented in the Inspyred Python library [16]) with a population composed of 200 individuals evolving during 20 generations. The sensitivity matrix $S$ is obtained using the tolerance analysis software AnaTole [14] and the non-conformity rate is evaluated with a Monte Carlo method implemented in OpenTURNS [17].

The non-dominated points obtained are displayed in Fig. 7Non-dominated points obtained after optimization, with four separate zones of iso-technique and varying allocated tolerancesfigure.1.7. The assembly process plan corresponding to the square point 
Table 2. List of the assembly techniques available associated to the joints they can be used for.

\begin{tabular}{lll}
\hline Index Name & Feasible joints \\
\hline 1 & Positioning with tool & $t j 3$ to $t j 7$ \\
2 & Positioning with adjustable tool & $t j 3$ to $t j 7$ \\
3 & Positioning with a robot as tool & $t j 3$ to $t j 7$ \\
4 & Positioning with a specific automated station $t j 3$ to $t j 7$ \\
5 & Back-to-back positioning & $t j 1$ and $t j 2$ \\
6 & Back-to-back positioning with splitting & $J 3$ and $J 5$ \\
7 & Traditional bonding & $J 1$ and $J 2$ \\
8 & Rapid bonding & $J 1$ and $J 2$ \\
9 & Drilling with grid and Manual fastening & $J 4$ and $J 6$ \\
10 & Drilling and Fastening with robot & $J 4$ and $J 6$ \\
11 & Drilling and Fastening with a specific station $J 4$ and $J 6$ \\
\hline
\end{tabular}

Table 3. Details of the technique Positioning with a robot as tool

\begin{tabular}{llllll}
\hline Technique name: & \multicolumn{5}{l}{ Positioning with a robot as tool } \\
Operations & Fixed cost & Duration & Resource $(s)$ & Quantity(ies) & Capabilities \\
\hline Robot referencing & 0 & 1 & Robot & 1 & \\
Component Grabbing & 0 & 0.5 & Robot & 1 & \\
Component Positioning & 0 & 0.5 & Robot & 1 & $\mathcal{U}(-0.07,0.07)$ \\
\hline
\end{tabular}

Resource hourly rate: Robot, 0.7 cost unit / $\mathrm{mn}$

Resource acquisition cost: Robot, $c_{\mathrm{nr}}=50000$ cost units

in Fig. 7Non-dominated points obtained after optimization, with four separate zones of iso-technique and varying allocated tolerancesfigure.1.7 is detailed in Table 4Assembly process plan corresponding to the square point in Fig. 7Non-dominated points obtained after optimization, with four separate zones of iso-technique and varying allocated tolerancesfigure.1.7table.1.4.

The results illustrate the interest of a multi objective optimization. Considering a single objective for the fitness and the other one as constraint would give an arbitrary boundary between acceptable and non-acceptable solution, leading to an optimal point not necessarily better than other ones. Here, a decision-making team can adapt the final choice with more information in hand.

A deeper analysis of the results displayed in Fig. 7Non-dominated points obtained after optimization, with four separate zones of iso-technique and varying allocated tolerancesfigure.1.7 shows that the points can be classified into four zones for this use case. In each zone, the assembly techniques selected are identical and only tolerances allocated are varying. It is therefore possible to identify the most relevant set of assembly techniques early during the design of the product and to refine the tolerance allocation along its design process. 


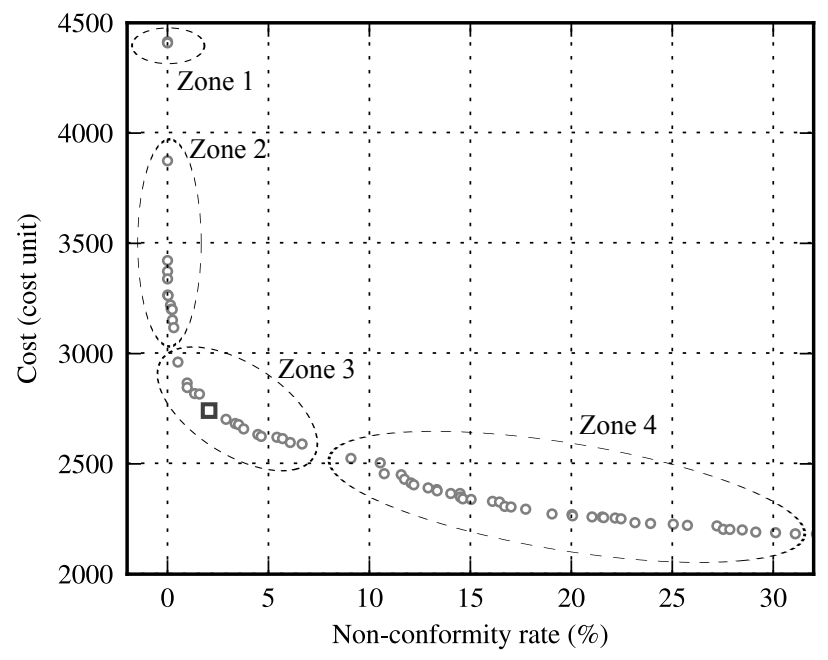

Fig. 7. Non-dominated points obtained after optimization, with four separate zones of isotechnique and varying allocated tolerances.

Table 4. Assembly process plan corresponding to the square point in Fig. 7Non-dominated points obtained after optimization, with four separate zones of iso-technique and varying allocated tolerancesfigure.1.7

\begin{tabular}{lllll}
\cline { 1 - 2 } Joint & Assembly technique selected & & \multicolumn{2}{c}{ Dimension Allocated tolerance } \\
\hline$t j 1$ and $t j 2$ & Back-to-back positioning & $L_{1}$ & $\mathcal{U}(-0.40,0.30)$ \\
$t j 3$ to $t j 7$ & Positioning with robot as a tool & $L_{2}$ & $\mathcal{U}(-0.40,0.30)$ \\
$J 1$ and $J 2$ & Traditional bonding & $L_{3}$ & $\mathcal{U}(-0.22,0.21)$ \\
$J 3$ and $J 5$ & Back-to-back positioning with splitting & $L_{4}$ & $\mathcal{U}(-0.17,0.16)$ \\
$J 4$ and $J 6$ & Drilling with grid and Manual Fastening & &
\end{tabular}

\section{Conclusion}

Selecting assembly techniques and allocating geometrical tolerances for a product requires making quality vs. cost trades-off. The method proposed in this paper helps the assembly process planner to find a set of $\operatorname{good}^{5}$ solutions among which he can select the one that suits the best his interests.

The method is based on an assembly technique library in which the company knowhow can be stored and upon a geometrical variation propagation relation associated to the assembly sequence. A wide range of potential assembly process plans can therefore be investigated and evaluated from an objective point of view. Results can be obtained with various assembly sequence scenarios and even with various product architecture scenarios. Decisions about the product's design and its assembly process plan can be taken from a point of view shared by product designers and manufacturers, enhancing collaborative and concurrent engineering.

\footnotetext{
${ }^{5}$ The non-dominated points, from the mathematical point of view.
} 
Acknowledgment. This research work has been carried out in partnership between EADS Innovation Works and the LURPA-ENS de Cachan in the frame of the GRCFlexible Assembly of the INNO'CAMPUS program.

\section{References}

1. Boothroyd, G., Dewhurst, P.: Product design for manufacture and assembly. Manufacturing Engineering 100, 42-46 (1988)

2. Wang, L., Keshavarzmanesh, S., Feng, H.Y., Buchal, R.: Assembly process planning and its future in collaborative manufacturing: a review. The International Journal of Advanced Manufacturing Technology 41, 132-144 (2009)

3. Bourjault, A.: Contribution à une Approche Méthodologique de L'Assemblage Automatisé: Élaboration Automatique des Sequences Opératoires. Ph.D. thesis, Universite de FrancheComte (1984)

4. De Fazio, T., Whitney, D.: Simplified generation of all mechanical assembly sequences. IEEE Journal of Robotics and Automation 3(6), 640-658 (1987)

5. Dini, G., Failli, F., Lazzerini, B., Marcelloni, F.: Generation of optimized assembly sequences using genetic algorithms. CIRP Annals - Manufacturing Technology 48(1), 17 - 20 (1999)

6. Homem de Mello, L., Sanderson, A.: A correct and complete algorithm for the generation of mechanical assembly sequences. IEEE Transactions on Robotics and Automation 7(2), 228-240 (1991)

7. Mantripragada, R., Whitney, D.: The datum flow chain: A systematic approach to assembly design and modeling. Research in Engineering Design 10(3), 150-165 (1998)

8. Cao, T., Sanderson, A.: Task decomposition and analysis of robotic assembly task plans using petri nets. Industrial Electronics, IEEE Transactions on 41(6), 620-630 (1994)

9. Becker, C., Scholl, A.: A survey on problems and methods in generalized assembly line balancing. European Journal of Operational Research 168(3), 694 - 715 (2006)

10. CIRP: Dictionnary of Production Engineering; Vol IV - Assembly (2012)

11. Abdullah, T.A., Popplewell, K., Page, C.J.: A review of the support tools for the process of assembly method selection and assembly planning. International Journal of Production Research 41(11), 2391 - 2410 (2003)

12. Camelio, J., Hu, S.J., Ceglarek, D.: Modeling variation propagation of multi-station assembly systems with compliant parts. Journal of Mechanical Design 125(4), 673-681 (2003)

13. Mounaud, M., Thiébaut, F., Bourdet, P., Falgarone, H., Chevassus, N.: Assembly sequence influence on geometric deviations propagation of compliant parts. International Journal of Production Research 49(4), 1021-1043 (2011)

14. Marguet, B., Chevassus, N., Falgarone, H., Bourdet, P.: Geometrical behavior laws for computer aided tolerancing: Anatole a tool for structural assembly tolerance analysis. In: 8th CIRP Seminar on Computer-Aided Tolerancing, Charlotte, NC USA (2003)

15. Chase, K., Greenwood, W., Loosli, B., Hauglund, L.: Least cost tolerance allocation for mechanical assemblies with automated process selection. Manufacturing Review 3(1), 4959 (1990)

16. Inspyred 1.0: Bio-inspired Algorithms in Python (May 2012), http://inspyred. github.com/, consulted on May 4, 2012

17. Open TURNS - Reference Guide - version 1.0 (April 2012), http: / / doc . openturns . org/openturns-1.0/openturns-doc\_april2012/pdf/OpenTURNS \

_ReferenceGuide.pdf 